\title{
DESENVOLVIMENTO E ANÁLISE SENSORIAL DE CHOCOLATES EM BARRA ADICIONADOS DE POLPA DE GABIROBA DESIDRATADA
}

Development and sensorial analysis of bar chocolates added from powder of dehydrated gabiroba

Desarrollo y análisis sensorial de chocolates en barra agregados de polpa de gabiroba desidratada

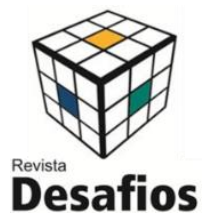

Sarah do Rezende do Nascimento ${ }^{* 1}$, Leonardo Rodrigues de Jesus ${ }^{1}$, Jéssyca Santos Silva ${ }^{1}$, Danilo José Machado de Abreu ${ }^{1}$, Clarissa Damiani ${ }^{1}$, Fernanda Salamoni Becker ${ }^{1}$

${ }^{1}$ Curso de Engenharia de Alimentos, Universidade Federal de Goiás, Goiânia, Brasil.

*Correspondência: Escola de Agronomia, Rodovia Goiânia-Nova Veneza, Km 0, s/n-Campus Samambaia, Goiânia, Goiás, Brasil. CEP: 74690900.e-mail sarah.renascim@gmail.com

Artigo recebido em 29/04/2019 aprovado em 03/05/2019 publicado em 16/06/2019.

\section{RESUMO}

A Gabiroba (Campomanesia spp.), um fruto nativo do cerrado, possui sabor adocicado, polpa suculenta sendo amplamente empregada na produção de doce, sorvete, licor e outros produtos. Objetivou-se, neste trabalho, utilizar a polpa de gabiroba no desenvolvimento de barras de chocolate, adicionando polpa desidratada de gabiroba, e verificar sua aceitação por meio da realização de análise sensorial. Foram elaboradas duas formulações de barras de chocolates, utilizando na primeira blend de chocolate ao leite e chocolate amargo, e na segunda chocolate branco. Realizou-se análise sensorial quantitativa, aplicando o teste de aceitação e intenção de compra a 105 provadores. Os resultados obtidos pelo cálculo do Índice de Aceitação (IA) mostraram que os atributos cor e aroma obtiveram maior IA para as barras de chocolate blend adicionadas de polpa de gabiroba desidratada. As barras de chocolate branco, adicionadas de polpa de gabiroba desidratada, obtiveram maior IA apenas para o atributo cor. Para a intenção de compra, a maior parcela dos provadores respondeu que "certamente compraria" ou "provavelmente compraria" as duas formulações. A adição de polpa de gabiroba desidratada em chocolates mostrou-se viável, sensorialmente, com bons percentuais de intenção de compra, indicando possível inclusão no mercado de um novo produto à base de gabiroba.

Palavras-chave: Campomanesia spp., Frutos do Cerrado, Teste de aceitação.

\section{ABSTRACT}

Gabiroba (Campomanesia spp.), a fruit of cerrado, having sweet flavor, juicy pulp and widely used in the production of sweets, ice cream, liquor and other products. The objective of this work was to use gabiroba pulp in the development of chocolate bars, adding dehydrated pulp of gabiroba, and to verify its acceptance by conducting sensorial analysis. Two formulations of bars of chocolates were elaborated, using in the first blend of milk chocolate and bitter chocolate, and in the second white chocolate. A quantitative sensory analysis was performed, applying the acceptance test and purchase intention to 105 testers. The results obtained by the calculation of the Acceptance Index (IA) showed that the color and aroma attributes obtained higher AI for the chocolate bars added with dehydrated gabioba pulp. White chocolate bars, added with dehydrated gabiroba pulp, obtained higher AI only for the color attribute. For the intention of purchase, the greater part of the tasters replied that "certainly would buy" or "probably would buy" the two formulations. The addition of dehydrated gabiroba pulp in chocolates proved to be viable, sensorially, with good percentages of purchase intention, indicating a possible inclusion in the market of a new gabiroba product.

Keywords: Campomanesia spp., Fruits of Cerrado, Acceptance test. 


\section{RESUMEN}

A Gabiroba (Campomanesia spp.), un fruto del cerrado, poseyendo sabor dulce, pulpa jugosa y ampliamente empleada en la producción de dulces, helados, licor y otros productos. En este trabajo, se objetivó utilizar la pulpa de gabiroba en el desarrollo de barras de chocolate, agregando pulpa deshidratada de gabiroba, y verificar su aceptación por medio de la realización de análisis sensorial. Se elaboraron dos formulaciones de barras de chocolates, utilizando en la primera mezcla de chocolate a la leche y chocolate amargo, y en el segundo chocolate blanco. Se realizó un análisis sensorial cuantitativo, aplicando la prueba de aceptación e intención de compra a 105 probadores. Los resultados obtenidos por el cálculo del Índice de aceptación (IA) mostraron que los atributos color y aroma obtuvieron mayor IA para las barras de chocolate blend añadidas de pulpa de gabardina deshidratada. Las barras de chocolate blanco, añadidas de pulpa de gabardina deshidratada, obtuvieron mayor IA sólo para el atributo color. Para la intención de compra, la mayor parte de los probadores respondió que "ciertamente compraría" o "probablemente compraría" las dos formulaciones. La adición de pulpa de gabiroba deshidratada en chocolates se mostró viable, sensorialmente, con buenos porcentajes de intención de compra, indicando posible inclusión en el mercado de un nuevo producto a base de gabiroba.

Descriptores: Campomanesia spp., Frutos del Cerrado, Prueba de aceptación.

\section{INTRODUÇÃO}

O Cerrado brasileiro abrange cerca de 2 milhões de $\mathrm{km}^{2}$ do Brasil Central, representando cerca de $23 \%$ da superfície terrestre do país. Em termos de área, é ultrapassado apenas pela floresta Amazônica (BRASIL, 2011). A região de cerrado estende-se desde a margem da floresta amazônica até as áreas periféricas, no sul dos estados de São Paulo e Paraná.

Com relação a flora do cerrado, esse possui diversas espécies frutíferas, com grande potencial de utilização agrícola, que são tradicionalmente aproveitadas pela população local. Os frutos, em geral, são consumidos in natura ou na forma de sucos, licores, sorvetes, geleias e doces diversos (ALMEIDA, 1998; SILVA et al., 2001), podendo também ser utilizados no desenvolvimento de produtos inovadores e saudáveis na indústria alimentícia, principalmente por serem frutas exóticas com características sensoriais únicas e intensas (MORZELLE et al.,2015).

A família Myrtaceae é composta por 145 gêneros e 5970 espécies, conhecidas espalhadas pelo mundo (THE PLANT LIST - MYRTACEAE, 2013), dentre as quais a espécie nativa brasileira é denominada Campomanesia xanthocarpa Berg. C. xanthocarpa, também, é popularmente conhecido como gabiroba (SARMENTO et al., 2012). Seus frutos podem ser consumidos in natura ou utilizados em formulações alimentícias (KINUPP e BARROS, 2008). Compostos de folhas de C.xanthocarpa e o extrato lipofílico de seus frutos, também, são conhecidos por suas propriedades medicinais: anti-inflamatório e antioxidante (KLAFKE et al., 2016), antiulcerogênicos (MARKMAN et al., 2004) e redução dos níveis de colesterol e obesidade (BIAVATTI et al., 2004).

A fruta da gabiroba é composta por $7 \%$ de cálice, $16 \%$ de sementes, $17 \%$ de casca e $60 \%$ de polpa (SANTOS et al., 2012). Os frutos podem ser considerados alimento funcional, devido às propriedades nutricionais, como alto teor de carboidratos (7,8 a 10,2\%), fibra alimentar (4,1 a 9,8\%), proteína (1,0 a 1,1\%) e lipídios (0,7 a 1,9\%). Além disso, os frutos possuem grande quantidade de água (79,1-83,5\%), vitamina C (826,26 mg g- 1$)$ e fenólicos totais $(19,59 \mu \mathrm{g}$ g- 1 ) (VALLILO et al., 2008; ANDRADE et al., 2012; SANTOS et al., 2012; EMBRAPA, 2015; BARBIERI et al., 2017). As propriedades nutricionais da polpa de gabiroba a tornam adequada ao consumo in natura e como matéria-prima com excelentes propriedades para aplicação na indústria de alimentos (CORADIN et al., 2011). No entanto, apesar do potencial de aplicação, a polpa da gabiroba não é utilizada comercialmente, 
podendo, portanto, ser utilizada no enriquecimento nutricional de produtos alimentícios, como por exemplo, os chocolates.

As categorias primárias de chocolate são conhecidas como escuro, ao leite e branco, que diferem, principalmente, no teor de sólidos de cacau, gordura do leite e manteiga de cacau presentes na formulação (AFOWAKA et al., 2008).

As características sensoriais, como o sabor, aroma, textura e cor dos produtos de chocolate são os principais critérios para determinar a aceitabilidade pelo consumidor. Produtos de chocolate com características de qualidade desejadas são amplamente consumidos por pessoas de todas as idades em todo o mundo (TOKER et al., 2018). O perfil sensorial é um método quantitativo que mede a intensidade dos atributos sensoriais de alimentos ou bebidas (MEILGAARD et al., 1991; STONE et al., 2004). Em condições controladas e, de acordo com um protocolo definido, um painel avalia os produtos, classificando cada uma das propriedades sensoriais em escalas de intensidade.

Diante do exposto, objetivou-se, com a realização deste trabalho, utilizar a polpa de gabiroba no desenvolvimento de um novo produto, elaborando duas formulações de chocolate em barra (blend $\mathrm{e}$ branco), assim como verificar sua aceitação por meio da análise sensorial.

\section{MATERIAIS E MÉTODOS}

\section{Material}

Para a elaboração das formulações dos chocolates em barra foram utilizados blend de chocolate $(50 \%$ chocolate ao leite $+50 \%$ chocolate amargo), chocolate branco, ambos da marca comercial
Garoto, adquiridas no comércio local da cidade de Goiânia, Goiás, Brasil.

A polpa da gabiroba foi doada congelada pela indústria processadora de gelados comestíveis "Frutos do Brasil”, localizada em Goiânia, Goiás, Brasil, em março de 2017.

\section{Métodos}

\section{Secagem da Polpa de Gabiroba}

A polpa de gabiroba foi, primeiramente, descongelada sob refrigeração à temperatura de $7{ }^{\circ} \mathrm{C}$, por um período de 12 horas e, posteriormente, desidratada em estufa à vácuo (modelo TE-395), acoplada por bomba de vácuo e pressão (modelo TE0581), ambos equipamentos da marca TECNAL Equipamentos Científicos (Brasil). A secagem da polpa aconteceu de forma gradativa, distribuindo-se uniformemente fina camada de polpa em bandejas de metal, as quais foram levadas à estufa durante, aproximadamente, 24 horas (6 horas com auxílio do vácuo e 18 horas com ausência de vácuo), havendo a necessidade de revolver a polpa de hora em hora, durante as 6 primeiras horas, para evitar fermentação e a formação de crosta que impossibilitasse a evaporação da água contida na polpa. Após a secagem, a polpa desidratada, apresentou-se em pequenos fragmentos, lembrando o aspecto de uva-passas. A polpa desidratada foi alocada em sacos plásticos e acondicionada em freezer $\left(-18^{\circ} \mathrm{C}\right)$.

\section{Formulação dos Chocolates em Barra}

As barras de chocolate foram elaboradas no Laboratório de Análise Sensorial de Alimentos, da Escola de Agronomia, da Universidade Federal de Goiás - UFG, em duas formulações diferentes, sendo a primeira com blend de chocolate amargo e chocolate ao 
leite, e a segunda com chocolate branco, ambas seguindo-se o fluxograma descrito na Figura 1.

Figura 1. Fluxograma do processamento de chocolates em barra adicionados de polpa de gabiroba desidratada.

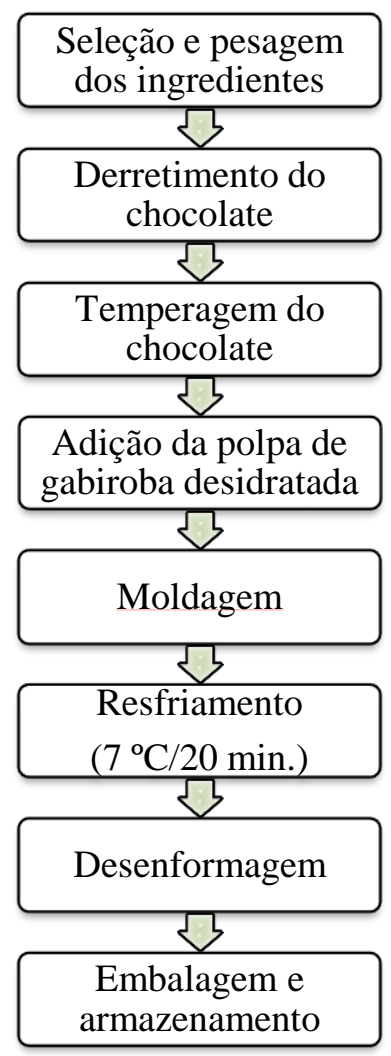

Os chocolates em barra foram formulados em bateladas de $1,1 \mathrm{~kg}$, na proporção de $1 \mathrm{~kg}$ de chocolate adicionados de $0,1 \mathrm{~kg}$ de polpa de gabiroba desidratada. O derretimento e a temperagem do chocolate ocorreram conforme indicação descrita no rótulo do produto, sendo o derretimento realizado em forno micro-ondas com potência média por um período de, aproximadamente, 5 minutos. Já a temperagem foi realizada colocando o refratário com o chocolate derretido em banho-maria frio, mexendo continuamente até atingir a temperatura aproximada de $29{ }^{\circ} \mathrm{C}$.

Após temperagem, adicionou-se, à massa de chocolate, fragmentos de polpa desidratada de gabiroba e a mistura foi homogeneizada, despejada em formas de policloreto de polivinila (PVC) com moldes retangulares nas dimensões de 2 x $3 \mathrm{~cm}$, retirando-se o excesso de chocolate das bordas com auxílio de espátulas de silicone e, em seguida, levando-se as formas ao refrigerador $\left(7^{\circ} \mathrm{C}\right)$, por um período de, aproximadamente, 20 minutos. Após resfriamento, as barras de chocolate, com polpa de gabiroba desidratada (Figura 5 e 6), com massa média de $6 \mathrm{~g}$, foram desenformadas, embaladas em papel metalizado e acondicionadas em refrigerador $\left(7^{\circ} \mathrm{C}\right)$ até a realização da avaliação sensorial, obtendo-se, assim, as amostras de chocolate em barra blend e branco adicionadas de polpa de gabiroba desidratada.

\section{Análise Sensorial}

A análise sensorial foi realizada no centro de eventos da Universidade Federal de Goiás, Professor Ricardo Freua Bufáiçal, durante o $14^{\circ}$ Congresso de Pesquisa, Ensino e Extensão (CONPEEX), no dia 17 de outubro de 2017.

Cada uma das duas formulações de chocolates em barra (blend e branco), adicionados de polpa desidratada de gabiroba, foi avaliada por 105 provadores não treinados, de ambos os sexos, com idades entre 18 e 69 anos, utilizando-se metodologia sensorial afetiva quantitativa, empregando-se escala hedônica com 9 pontos, sendo o valor máximo 9 (gostei muitíssimo) e o mínimo (desgostei muitíssimo), analisando-se os atributos sensoriais de avaliação global, cor, aroma, textura e sabor (DUTCOSKY, 2015). A seleção de provadores deu-se, apenas, por meio de questionamento informal, imediatamente antes da realização do teste sensorial, procurando-se saber da apreciação de chocolate, por parte dos possíveis provadores, sendo dispensados da análise sensorial provadores que afirmaram não gostar deste tipo de produto. 
Computou-se a frequência de consumo de chocolate em barra, segundo seis diferentes classes de consumo: diariamente, 3 vezes por semana, 1 vez por semana, quinzenalmente, mensalmente e não consome. Já a intenção de compra dos chocolates em barra, adicionados de polpa de gabiroba desidratada, foi obtida utilizando-se escala hedônica de 5 pontos, sendo o valor mínimo 1 (certamente compraria) e o máximo 5 (certamente não compraria) (DUTCOSKY, 2015).

As amostras de chocolate em barra blend e branco, adicionados de polpa de gabiroba desidratada, foram codificadas com números aleatórios de três (3) dígitos e servidas aos provadores de forma monádica e aleatória, a temperatura ambiente. Para minimizar possíveis efeitos residuais (carry over), foram servidos, juntamente com as amostras, água mineral natural para ser utilizado na limpeza do palato entre as avaliações de uma amostra e outra (MEILGAARD et al., 2015).

Os provadores receberam uma ficha de avaliação na qual solicitava-se que atribuíssem notas de 1 a 9, para cada amostra codificada, conforme escala hedônica; além de indicarem a frequência de consumo de chocolate e a intenção de compra do produto avaliado.

A partir das notas obtidas para os atributos avaliados, em cada uma das formulações desenvolvidas, calculou-se o índice de aceitabilidade, conforme equação 1 :

$$
\mathrm{IA}=\frac{A}{B} * \mathbf{1 0 0}
$$

Onde: IA = Índice de Aceitabilidade em \%;
A = Escore da escala hedônica médio obtido pelo atributo;

$\mathrm{B}=$ Escore da escala hedônica máximo dado ao atributo;

\section{Análise Estatística}

Foi realizada análise descritiva do perfil dos provadores e obtido a média dos descritores sensoriais. Todas as análises estatísticas foram obtidas pelos softwares R-project (R Development Core Team, 2017) e Microsoft Excel® 2013.

\section{Dados Comitê de Ética}

Número do parecer: 502.167; Data da relatoria: 16/12/2013.

\section{RESULTADOS E DISCUSSÃO}

Os resultados obtidos no teste de aceitação, para os atributos avaliação global, cor, aroma, textura e sabor, para as duas formulações de chocolates em barra, adicionados de polpa de gabiroba desidratada, são demonstrados na Tabela 1.

Todos os atributos analisados obtiveram escores superior a 7 (gostei moderadamente) para ambas formulações de barra de chocolate adicionados de polpa de gabiroba desidratada. A cor foi o atributo que apresentou os maiores escores, superior a 8 (Gostei muito), tanto para as barras de chocolate blend, quanto para as barras de chocolate branco. $\mathrm{O}$ atributo aroma apresentou escore igual a 8 (Gostei muito) para o produto elaborado com o chocolate blend. Com essas médias obtidas, acima de 7 , pode-se dizer que ambas as formulações foram apreciadas pelos provadores. 
Tabela 1. Médias de notas atribuídas pelos provadores na avaliação sensorial de barras de chocolate blend e chocolate branco com polpa de gabiroba desidratada

\begin{tabular}{cccccc}
\hline \multirow{2}{*}{ Tipo } & \multicolumn{5}{c}{ Atributos* } \\
\cline { 2 - 6 } & AG & Cor & Aroma & Textura & Sabor \\
\hline Blend & 7,7 & 8,1 & 8,0 & 7,4 & 7,5 \\
Branco & 7,6 & 8,1 & 7,5 & 7,6 & 7,3 \\
\hline
\end{tabular}

* Classificação hedônica: 1 “desgostei muitíssimo“ a 9 “gostei muitíssimo“; AG: Avaliação Global.

Resultados semelhantes foram encontrados por

Fritsch et al. (2015) que estudaram a adição de guaraná em pó em chocolates em barra, obtendo para a formulação adicionada de $6 \%$ de guaraná em pó escores de 7,97 para o atributo aroma, 7,47 (equivalente a "gostei regularmente" pela escala hedônica utilizada pelo autor) para o atributo sabor e 8,17 (equivalente a "gostei muito" pela escala hedônica utilizada pelo autor) para o atributo textura. Carvalho (2016), também, encontrou resultados semelhantes quando adicionou uva em pó a barras de chocolate ao leite, quando obteve maior quantidade de escores 8 (equivalente a "gostei muito" pela escala hedônica utilizada pelo autor) tratando-se de sabor; escores entre 7 (equivalente a "gostei regularmente" pela escala hedônica utilizada pelo autor) e 9 (equivalente a "gostei muitíssimo" pela escala hedônica utilizada pelo autor) para o atributo textura e novamente escores 8 , tratandose da avaliação global do produto.

Na Tabela 2 são apresentados os índices de aceitabilidade, obtidos para cada um dos atributos das duas formulações desenvolvidas.

Tabela 2. Índice de aceitabilidade (\%) dos atributos de barras de chocolate blend e chocolate branco com polpa de gabiroba desidratada.

\begin{tabular}{cccccc}
\hline \multirow{2}{*}{ Tipo } & \multicolumn{5}{c}{ Atributos $(\%)$} \\
\cline { 2 - 6 } & AG & Cor & Aroma & Textura & Sabor \\
\hline \multirow{2}{*}{ Blend } & 85,6 & 90,0 & 88,9 & 82,2 & 83,3 \\
Branco & 84,4 & 90,0 & 83,3 & 84,4 & 81,1 \\
\hline
\end{tabular}

AG: Avaliação Global.

Os resultados obtidos pelo cálculo do índice de aceitabilidade (IA) indicaram que todos os atributos analisados apresentaram IA maior que $80,0 \%$. As barras de chocolate tipo blend, adicionadas de polpa de gabiroba desidratada, obtiveram maior IA para os atributos cor e aroma, 90,0\% e 88,8\% respectivamente de aceitabilidade. Já as barras de chocolate tipo branco, adicionadas de polpa de gabiroba desidratada, apresentaram maior IA somente para o atributo cor, 90 $\%$ de aceitabilidade. Dutcosky (2015), constatou que
IA $\geq 70 \%$ representa que o produto desenvolvido terá um bom resultado na aceitação pelo público consumidor. Portanto, as duas formulações desenvolvidas apresentam grande chance de serem aceitas pelos consumidores.

Em relação a idade e o sexo, os provadores apresentaram idade entre 18 e 69 anos (Figura 2), sendo $71 \%$ do sexo feminino e $29 \%$ masculino. 
Figura 2. Faixa etária de provadores participantes na avaliação sensorial de barras de chocolate blend e branco adicionados de polpa de gabiroba desidratada.

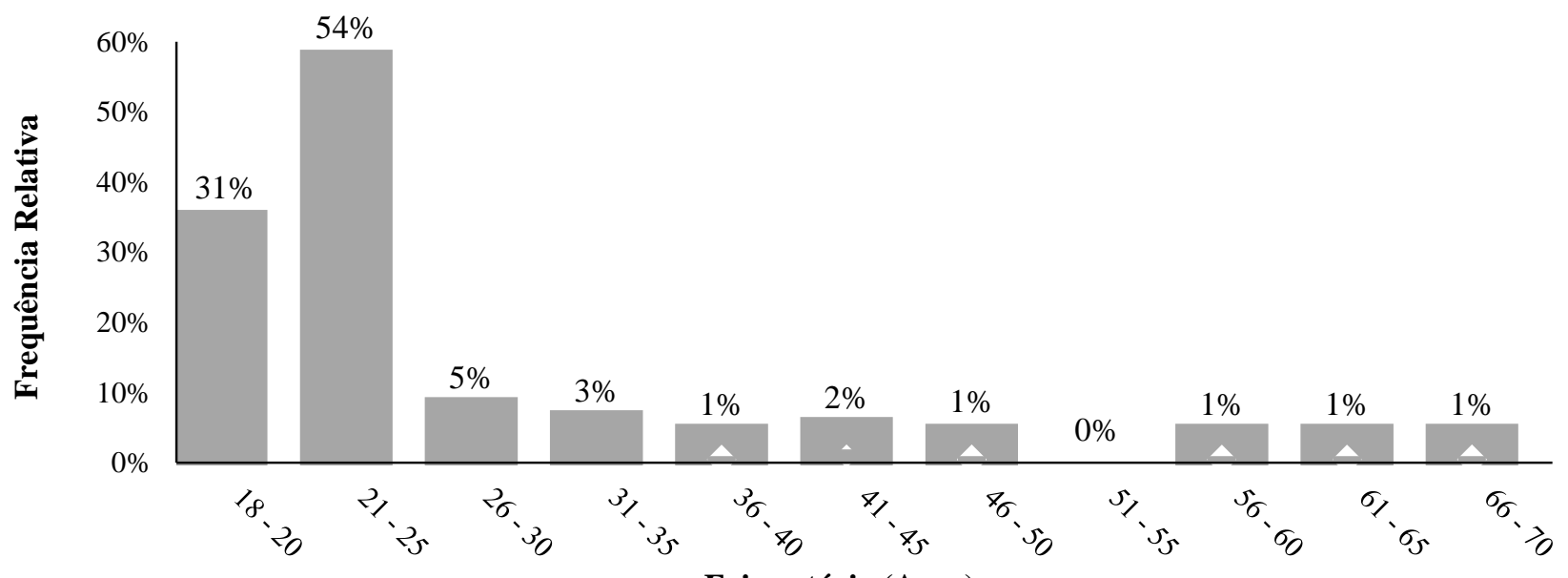

Faixa etária (Anos)

A maioria dos provadores $(85,0 \%)$ apresentaram faixa etária entre 18 e 25 anos. Apenas $15 \%$ dos provadores apresentaram idade superior a 25 anos. Tal comportamento pode ser atribuído ao fato da análise sensorial ter sido realizada em um evento voltado para a comunidade universitária. Segundo dados do Instituto Nacional de Estudos e Pesquisas Educacionais Anísio Teixeira (INEP, 2017), os discentes matriculados em instituições federais de ensino superior, no estado de Goiás, com idade entre 18 e 25 anos representam 70,3\% do total de alunos matriculados nessas instituições, fato este que explica a maioria dos provadores deste estudo apresentarem tal faixa etária.

Na Figura 3 estão apresentadas as frequências de consumo de chocolate dos provadores que participaram da análise sensorial das barras de chocolate blend e branco adicionados de polpa de gabiroba desidratada.

Com os resultados obtidos para a frequência de consumo de chocolate pelos provadores foi possível observar que $64,0 \%$ do total de provadores admitiram consumir chocolate pelo menos uma vez na semana e apenas $2,0 \%$ disseram que não consomem chocolate. Com isso, pode-se notar que o consumo de chocolate está em alta. Segundo dados da ABICAB (2015), o consumo anual per capita de chocolate no Brasil, no ano de 2014, foi de $2,5 \mathrm{~kg}$.

Figura 3. Frequência de consumo de chocolate dos provadores participantes na avaliação sensorial de barras de chocolate blend e branco adicionados de polpa de gabiroba

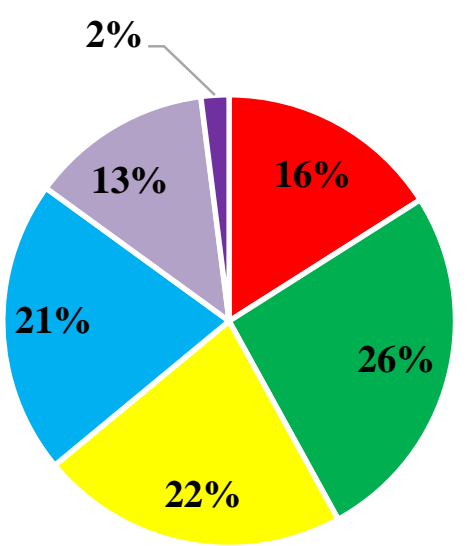

$$
\begin{array}{ll}
- \text { Diariamente } & -3 \text { vezes por semana } \\
-1 \text { vez por semana } & =1 \text { vez a cada } 15 \text { dias } \\
-1 \text { vez por mês } & - \text { Não consome }
\end{array}
$$


Dias e Gonçalves (2009) verificaram que, de um total de 100 indivíduos adultos, constituído por maioria do sexo feminino, cerca de $45,0 \%$ declararam consumir chocolate pelo menos uma vez na semana, quando tal frequência era mensal o percentual de indivíduos que declaravam consumir chocolate aumentava para $57,3 \%$.

Segundo Braga, Daolio e Oliveira (2007), há vários motivos que levam as pessoas a consumirem chocolate, dentre eles o sabor, o vício pelo produto, por ser prazeroso, para ajudar na digestão ou fornecer energia, por ansiedade ou por problemas emocionais especialmente a Tensão Pré-Menstrual (TPM) que afeta diretamente o comportamento feminino. Por esse motivo, muitas mulheres apontam que este fator de stress está vinculado ao consumo de chocolate.

Nas Figuras 4 e 5 estão apresentados os resultados para o teste de intenção de compra para cada formulação.

Com os resultados de intenção de compra, verifica-se que $67,0 \%$ dos provadores certamente ou provavelmente comprariam as barras de chocolate tipo blend, adicionado de polpa de gabiroba desidratada. Já as barras de chocolate branco, adicionado de polpa de gabiroba desidratada, obtiveram $62,0 \%$ dos

Figura 4. Intenção de compra das barras de chocolate blend adicionados de polpa de gabiroba desidratada

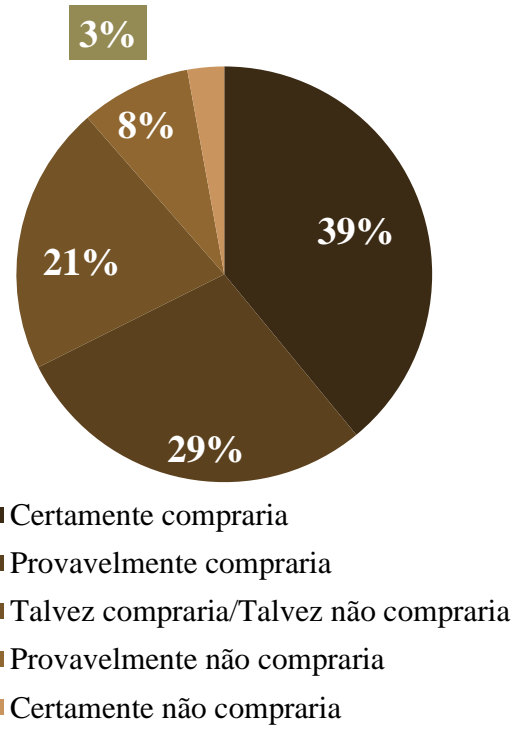

provadores que certamente ou provavelmente comprariam.

Figura 5. Intenção de compra das barras de chocolate branco adicionados de polpa de gabiroba desidratada.

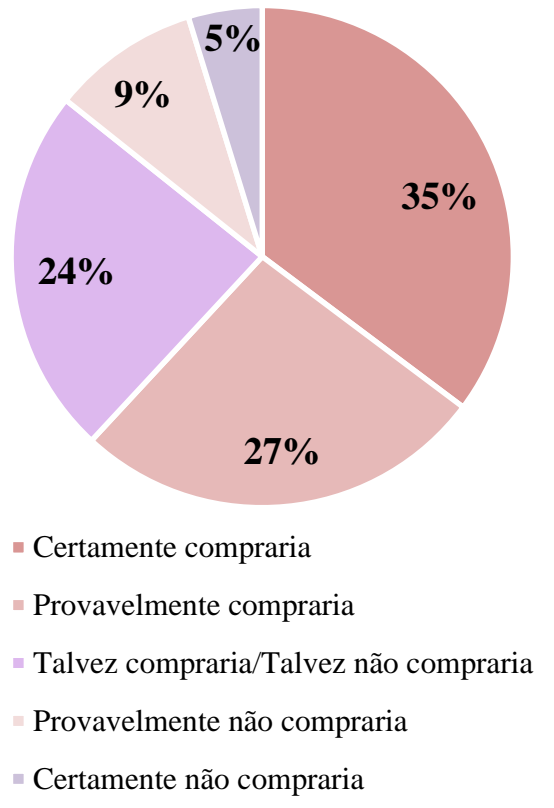

\section{CONCLUSÃO}

Assim sendo, foi possível desenvolver duas formulações de barras de chocolate com adição de $10 \%$ de polpa de gabiroba desidratada. De acordo com os testes sensoriais realizados, ambas as formulações de chocolate em barra, adicionadas de polpa de gabiroba desidratada, mostram-se viáveis sensorialmente, e bons percentuais de intenção de compra, indicando possível inclusão no mercado de um novo produto à base de gabiroba.

\section{AGRADECIMENTOS}

Á Coordenação de Aperfeiçoamento de Pessoal de Nível Superior (CAPES), Conselho Nacional de Desenvolvimento Científico e Tecnológico (CNPq) e a Universidade Federal de Goiás (UFG).

Todos os autores declararam não haver qualquer potencial conflito de interesses referente a este artigo. 


\section{REFERÊNCIAS}

ABICAB. Associação Brasileira da Indústria de Chocolate, Amendoim, Balas e Derivados -. Pesquisa e Estatísticas, 2015. Produção, consumo aparente, exportação e importação. Disponível em: http://www.abicab.org.br/estatisticas/. Acesso em: $11 / 06 / 2018$.

AFOWAKA, E.O.; PATERSON, A.; FOWLER, M.; VIEIRA, J. Effects of tempering and fat crystallization behavior on microstructure, mechanical properties and appearance in dark chocolate systems. Journal of Food Engineering. v.89, p.128-136, 2008.

ALMEIDA, S.P. Cerrado - Aproveitamento Alimentar. Planaltina: Embrapa-CPAC, 1998. 188p. Disponível em: http://www.cpac.embrapa.br/publicacoes/search_pbl/ 1?q=Aproveitamento\%20alimentar. Acesso em: 10/06/2018.

ANDRADE, D.R.M., HELM, V.M., MAZZA, A.M., MAZZA, M.C.M. Caracterização por composição nutricional da guabiroba. In: XXII Congresso Brasileiro de Fruticultura, Bento Gonçalves, Anais. p.5050-5053, 2012

BARBIERI, S.F., RUTHES, A.C., PETKOWICZ, C.L. DE O., DE GODOY, R.C.B., SASSAKI, G.L., SANTANA-FILHO, A., SILVEIRA, J.L.M. Extraction, purification and structural characterization of a galactoglucomannan from the gabiroba fruit (Campomanesia xanthocarpa Berg), Myrtaceae family. Carbohydrate Polymers. v. 174, p. 887-895, 2017.

BIAVATTI, M.W.; FARIAS, C.; CURTIUS, F.; BRASIL, L.M.; HORT, S.; SCHUSTER, L. Preliminary studies on Campomanesia xanthocarpa (Berg.) and Cuphea carthagenensis (Jacq.) J.F. Macbr. Aqueous extract: weight control and biochemical parameters. Journal of Ethnopharmacology. v.93, n.2-3, p.385-389, 2004.

BRAGA, C.; DAOLIO, R.; OLIVEIRA, M.A. O que motiva e determina a compra e o consumo de chocolate. Sociedade Brasileira de Estudos Interdisciplinares de Comunicação, n. 1, p. 1-10, 2007.

BRASIL. 2011. Monitoramento do desmatamento nos biomas brasileiros por satélite acordo de cooperação técnica MMA/IBAMA - Monitoramento do Bioma Cerrado 2009-2010. Brasília, agosto, 65 p.

CARVALHO, J.C.S. Desenvolvimento de chocolates ao leite com propriedades funcionais acrescidos de folhas de Brassica oleracea (couve) e fruto de Vitis vinefera (uva). São Paulo, SP. Dissertação de Mestrado. Universidade de São Paulo; 2016.

CORADIN, L.; SIMINSKI A.; REIS A. Espécies nativas da flora brasileira de valor econômico atual ou potencial: plantas para o futuro: Região Sul, Brasília: Ministério do Meio Ambiente. p. 159-162, 2011.

DIAS, J.R.; GONÇALVES, E.C.B.A. Avaliação do consumo e análise da rotulagem nutricional de alimentos com alto teor de ácidos graxos trans. Ciência e Tecnologia de Alimentos. v.1, n.29, p.177182, 2009.

DUTCOSKY, S. D. Análise sensorial de alimentos. 4.ed., Curitiba, Champagnat, 2015.

EMBRAPA. 2015. Valor nutricional da guabiroba. Disponível em:

https://www.embrapa.br/florestas/buscadepublicacoes/-/publicacao/1027135/valornutricional da-guabiroba. Acesso em: 12/06/2018.

FRITSCH, F.C.; SILVA, M.S.; DEGÁSPARI, C.H. Desenvolvimento e análise sensorial de formulações de chocolate em barra adicionadas de guaraná em pó. Cadernos da Escola de Saúde. v. 1, n. 13, p. 52-78, 2015.

INEP - Instituto Nacional de Estudos e Pesquisas Educacionais Anísio Teixeira. 2017. Sinopse Estatística da Educação Superior 2016. Brasília: Inep, $2017 . \quad$ Disponível em: http://portal.inep.gov.br/web/guest/sinopsesestatisticas-da-educacao-superior. Acesso em: 15/06/2018.

KINUPP, V.F., BARROS, I.B.I.D. Teores de proteína e minerais de espécies nativas, potenciais hortaliças e frutas. Ciência e Tecnologia de Alimentos. v. $28, \mathrm{n}$. 4, p. 846-857, 2008.

KLAFKE, J.Z.; PEREIRA, R.L.D.; HIRSCH, G.E.; PARISI, M.M.; PORTO, F.G.; ALMEIDA, A.S. Study of oxidative and inflammatory parameters in LDLr-KO mice treated with a hypercholesterolemic diet: Comparison between the use of Campomanesia xanthocarpa and acetylsalicylic acid. Phytomedicine. v.23, n.11, p.1227-1234, 2016.

MARKMAN, B.E.O., BACCHI, E.M., KATO, E.T.M. Antiulcerogenic effects of Campomanesia xanthocarpa. Journal of Ethnopharmacology. v.94, n.1, p.55-57, 2004.

MEILGAARD, M.C.; CIVILLE, G.V.; CARR, T. Sensory Evaluation Techniques. 5.ed. CRC Press, 2015. 
MEILGAARD, M.C., CIVILLE, G.V., CARR, B.T. Sensory evaluation techniques. 2 ed., CRC Press, 1991.

MORZELLE, M.C.; BACHIEGA, P.; SOUZA, E.C.; VILAS BOAS, E.V.B.; LAMOUNIER, M.L. Caracterização química e física de frutos de curriola, gabiroba e murici provenientes do Cerrado brasileiro Revista Brasileira de Fruticultura. v. 37, n. 1, p. 96103, 2015.

SANTOS, M.S., CORREIA, C.H., PETKOWICZ, C.L. DE O., CÂNDIDO, L.M.B. Evaluation of the technological potencial of gabiroba (Campomanesia xanthocarpa Berg) fruit. Journal of Nutritional \& Food Sciences. n.2, p. 2-9, 2012.

SARMENTO, M.B., CAROLINA, A., SANTOS, C. Recursos genéticos de frutas nativas da família Myrtaceae no Sul do Brasil. Magistra. v. 24, p. 250$262,2012$.

SILVA, D.B.; SILVA, J.A.; JUNQUEIRA, N.T.V.; ANDRADE, L.R.M. de Frutas do Cerrado. Brasília: Embrapa Informação Tecnológica. 2001. 179p.
STONE, H., SIDEL, J., OLIVER, S., WOOLSEY, A, SINGLETON, R.C. Sensory evaluation by quantitative descriptive analysis. Descriptive Sensory Analysis in Practice, p. 23-34, 2004.

THE PLANT LIST - Myrtaceae, 2013. Disponível em:

http://www.theplantlist.org/1.1/browse/A/Myrtaceae/. Acesso em: 06/06/2018.

TOKER, O.S.; KONAR, N.; PIROUZIAN, H.R.; OBA, S.; POLAT, D.G; PALABIYIK, I.; POYRAZOGLU, E.S.; SAGDIC, O. Developing functional white chocolate by incorporating diferente forms of EPA and DHA-Effects on product quality. LWT-Food Science and Technology. v.87, p.177 $185,2018$.

VALLILO, M.I., MORENO, P.R.H., OLIVEIRA, E., LAMARDO, L.C.A., GARBELOTTI, M.L. Composição química dos frutos de Campomanesia xanthocarpa Berg-Myrtaceae. Ciência e Tecnologia de Alimentos. v.28, n.0, p.231-237, 2008. 